\title{
Influence of anatomical barriers on maxillary incisor root resorption after orthodontic treatment with premolar extractions
}

Antonio Geraldo de Oliveira', Fabiana Guilhermina Ferreira Castro²

Intoduction: Apical root resorption is a frequent and occasionally critical problem in orthodontic patients undergoing induced tooth movement. One of the factors that might influence prognosis, especially in maxillary incisors, which most frequently present resorptions, are the so-called the anatomical barriers; that is, proximity of the buccal and palatal cortical bones to the maxillary incisor roots.

Objective: The purpose of this research was to investigate whether patients with excessive vertical growth really present a small distance between the alveolar cortical bones and the maxillary incisor roots, and whether there is a correlation between this distance and the root resorption index in comparison with patients presenting horizontal growth.

Methods: The sample comprised orthodontic records of 18 patients with extraction planning of first maxillary premolars and treatment by the standard and/or preadjusted edgewise brackets. Their initial and final periapical radiographs were evaluated to determine the amount of root resorption that occurred.

Results: On the palatal side, patients with excessive vertical growth (Group 2 - SN-GoGn $\geq 43^{\circ}$ ) showed a narrower alveolar bone than the horizontal growth patients (Group $1-\mathrm{SN}-\mathrm{GoGn} \leq 29^{\circ}$ ). However, the distance between the buccal cortical bone and the central incisor root apex showed no significant difference between Groups 1 and 2 ;

Conclusions: It was concluded that there are no correlations between the proximity of buccal cortical bone, maxillary incisor roots and the root resorption index.

Keywords: Anatomical barriers. Root resorption. Maxillary incisors. Orthodontic treatment.

${ }^{1}$ MSc in Orthodontics, Faculty of Dentistry of Bauru/USP. PhD in Orthodontics, São Leopoldo Mandic Faculty, Campinas.

${ }^{2}$ Specialist in Orthodontics, UNILAVRAS.

\author{
How to cite this article: Oliveira AG, Castro FGF. Influence of anatomical barri- \\ ers on maxillary incisor root resorption after orthodontic treatment with premolar \\ extractions. Dental Press J Orthod. 2012 Mar-Apr;17(2):77-82. \\ Submitted: August 22, 2008 - Revised and accepted: March 13, 2009 \\ " The authors report no commercial, proprietary, or financial interest in the products \\ or companies described in this article. \\ Contact address: Antonio Geraldo de Oliveira \\ Av. Rui Barbosa, 385 Sala 502 - Centro - CEP: 37002-140 - Varginha / MG - Brazil \\ E-mail: antoniogeraldooliveira@gmail.com
}




\section{INTRODUCTION}

Dental resorptions are tissue damages caused by immunopathological responses resulting from tooth injuries. This may be due to trauma, lesion, extensive dental displacements, orthodontic treatment with tooth extractions, prolonged orthodontic treatments with continuous force, use of inter-maxillary elastics, among other factors.

It is understood that the etiology of root resorption of orthodontically treated patients refers to local factors, such as the morphology of the root and alveolar bone crest. In response to the applied forces, each type of root reacts in a different manner. Therefore, one can predict which tooth will be susceptible to resorption during induced movement, by means of previous analysis of its periapical radiographs. ${ }^{2}$

The quantity of root movement, long roots, narrow roots, abnormal root shape, and the use of Class II elastics appear to be significant risk factors for root resorption. However, no association was found between the type of initial malocclusion, treatment time, use of rectangular wires, root proximity to the palate and root resorption. ${ }^{6}$

Thin alveolar bone can be found in both the buccal and lingual regions of mandibular incisors in patients presenting Class I, II and III malocclusions, with high SN-MP angle, and in a group of Class II individuals with a normal SN-MP angle. Thin alveolar bone may also be found in the lingual region of maxillary incisors in the Class II group with a high SN-MP angle. ${ }^{4}$

In the absence of a history of trauma or resorption before treatment, the dentoalveolar structure cannot be used to identify persons who would be predisposed to a large amount of apical root resorption during orthodontic treatment. ${ }^{8}$

Recently, contact of the root with buccal or palatal cortical bone at the root apex level during an orthodontic movement was related to root resorption. Dentofacial morphology (tooth contact with the cortical bone) was suggested as predisposing certain people to root resorption. Approximation of the root to the palatal cortical bone during orthodontic treatment explains $12 \%$ of root resorptions, and maxillary alveolar bone width accounts for approximately $2 \% .^{5}$

There are no statistically significant anatomical differences at the level of alveolar bone in the anterior region among dolichocephalic and brachycephalic in- dividuals with different types of malocclusions. ${ }^{1}$

In a study with the purpose of assessing whether the alveolar bone shape and its density could promote apical root resorption, it was demonstrated that the dentoalveolar complex dimensions remained relatively unaltered during tooth movement. The amount of alveolar bone around the root and densities of cortical and trabecular bone showed no significant correlation with the extent of external apical root resorption. ${ }^{7}$

Several factors favor predictability for dental resorption in orthodontic treatment, with emphasis on the morphology of roots and alveolar bone crest, previous history of dental trauma progression, aspects related to the distribution of forces, extent of movement, indication of dental extractions and the use of intermaxillary elastics. ${ }^{2}$

The thickness between the alveolar process of the maxilla and mandible and the patient's age, do not seem to be correlated, but type of malocclusion is dependent on the thickness of the buccal bone in the anterior region of the maxilla. Patients with Class III malocclusion present a higher percentage of buccal bone reduction in the anterior region of the maxilla when compared with Class II patients. Patients who have a tendency toward vertical growth present reduced dimension of the lingual bone of the maxilla and buccal bone of the mandible. ${ }^{3}$

Therefore, the aim of this study was to investigate whether patients with excessively vertical growth indeed have greater proximity of the cortical alveolar bones to the roots of maxillary incisors, in comparison with patients with horizontal growth, and whether there is correlation between this proximity and the rate of root resorption of these teeth.

\section{MATERIAL AND METHODS Material}

Orthodontic records of 456 patients who were treated at the Department of Orthodontics of the University Center of Lavras were evaluated. The sample was comprised of cases treated with maxillary first premolar extractions and with standard and pre-adjusted edgewise brackets.

Initial and final periapical radiographs of 18 patients were assessed to measure the amount of root resorption. Initial and final lateral cephalograms 
were also used to analyze the patients growth patterns (vertical or horizontal), based on determination of the SN-GoGn angle, and to evaluate the proximity of the buccal and palatal cortical bone to the roots of maxillary incisors.

\section{Methods}

To evaluate the quantity of apical root resorption, a caliper Model DEMEO (Norfol, reading accuracy, $0.1 \mathrm{~mm}$ ) was used to measure the length of maxillary central incisors in the patients' initial and final periapical radiographs, taken from the root apex up to the incisal edge, along the long axis of the tooth. The quantity of root resorption was obtained by subtracting the value of the final tooth length from the initial tooth length (Fig 1).

Manual cephalometric tracing was used. On each of the initial and final cephalograms, its respective tracing was delimited, which offers a set of variables that allow linear and angular measurements of craniofacial structures to be made for the purpose of research.

By means of cephalometry, the anterior and posterior alveolar thickness to the apex of the maxillary incisors was determined.

\section{Lines and planes (Fig 2)}

» SN line: Line that cross through points $\mathrm{S}$ and $\mathrm{N}$, situated in the midsagittal plane and at the base of the skull, and extends from one side to the other of the ultraphan paper. The SN line corresponds to the upper measurable limit of the cephalogram.

» Line A Perp: This line is perpendicular to the palatal plane passing through point $\mathrm{A}$. It begins in the palatal plane and ends $5 \mathrm{~mm}$ below the edge of the maxillary central incisor.

» Long axis of the maxillary central incisor: It begins at SN line, cross through the long axis of the tooth and ends $5 \mathrm{~mm}$ below the incisor edge.

» Mandibular plane (GoGn): Used by Riedel and Steiner. It represents the mandibular body more precisely than the points taken arbitrarily of the most inferior part of the mandible.

» Palatal plane: It starts on the ANS (anterior nasal spine), cross through point PNS (posterior nasal spine) and extends up to the margin of the ultraphan paper.

\section{Angles (Fig 3)}

» $\mathrm{SN}-\mathrm{GoGn}$ angle: Angle formed by the $\mathrm{SN}$ line and the GoGn mandibular plane. Considering that GoGn represents the base of the mandibular body, this angle elucidates the behavior of the mandibular base in relation to the base of the skull.

» Angle 1.PP: Angle formed by the long axis of the maxillary central incisor and the palatal plane.

\section{Measurements used in the study (Fig 3)}

» AAB (Anterior Anatomical Barrier): Bone anterior (buccal) to the apex of the maxillary incisor. This measurement goes from the buccal cortical bone to the apex of the maxillary incisor; perpendicular to the Line A perp.

» $\mathrm{PAB}$ (Posterior Anatomical Barrier): Bone posterior (palatal) to the apex of the maxillary incisor. This measurement goes from the apex of the maxillary incisor to the palatal cortical bone; perpendicular to the long axis of this incisor.

» $\mathrm{SN}-\mathrm{GoGn}$ : Angle formed by the $\mathrm{SN}$ line and the GoGn mandibular plane. Measured in the patient's initial cephalogram.

»1.PP: Angle formed by the line of the maxillary central incisor long axis with the palatal plane (ANS - PNS).

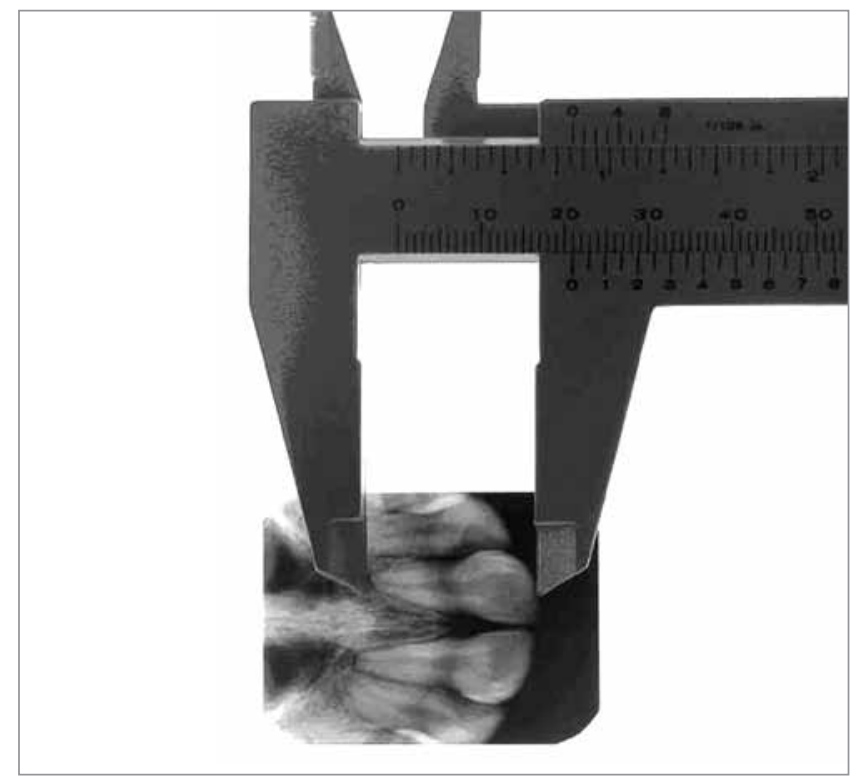

Figure 1 - Measurement of the maxillary central incisors. Caliper measuring the tooth length. 


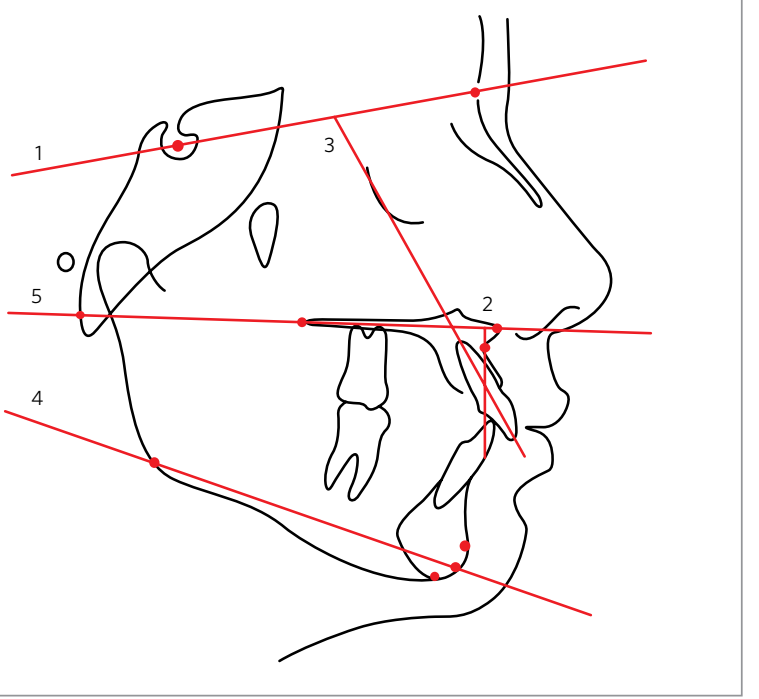

Figure 2 - Lines and planes used in the study. 1- SN line; 2- A perp line; 3Long axis of the maxillary central incisor; 4- Mandibular plane(GoGn); 5- palatal plane.

\section{RESULTS}

To study the influence of "Anatomical Barriers" in root resorption, it was necessary to first determine groups that would - theoretically - present anatomically narrower alveolar bones, which would be the true anatomical barriers. In this study, the option was to make this analogy in the region of the maxillary central incisors. Starting with this premise, the patients in the sample were divided into two groups, using the facial growth pattern as the criterion.

Thus, patients in Group 1, who presented horizontal growth, had an $\mathrm{SN}-\mathrm{GoGn}$ angle smaller than or equal to $29^{\circ}$. On the other hand, patients in Group 2, as opposed to Group 1, presented vertical craniofacial growth (angle $\mathrm{SN}-\mathrm{GoGn}$ greater than or equal to $43^{\circ}$ ) (Table 1 ).

In this study, the distance from the posterior alveolar bone to the apex of maxillary central incisor was statistically significant between Groups 1 and 2; that is, patients with excessively vertical growth (Group 2) had a much narrower alveolar bone in the lingual region of the maxillary incisors than patients who presented horizontal growth (Group 1). Whereas the distance from the buccal cortical bone to the apex of maxillary central incisor showed no significance for Groups 1 and 2 , which was not expected, since both groups presented quite distinct SN-GoGn angles (Table 2).

In Table 3 it can be noted that in both, Group 1 (SNGoGn $\left.\leq 29^{\circ}\right)$ and Group $2\left(\mathrm{SN}-\mathrm{GoGn} \geq 43^{\circ}\right)$, the crowns

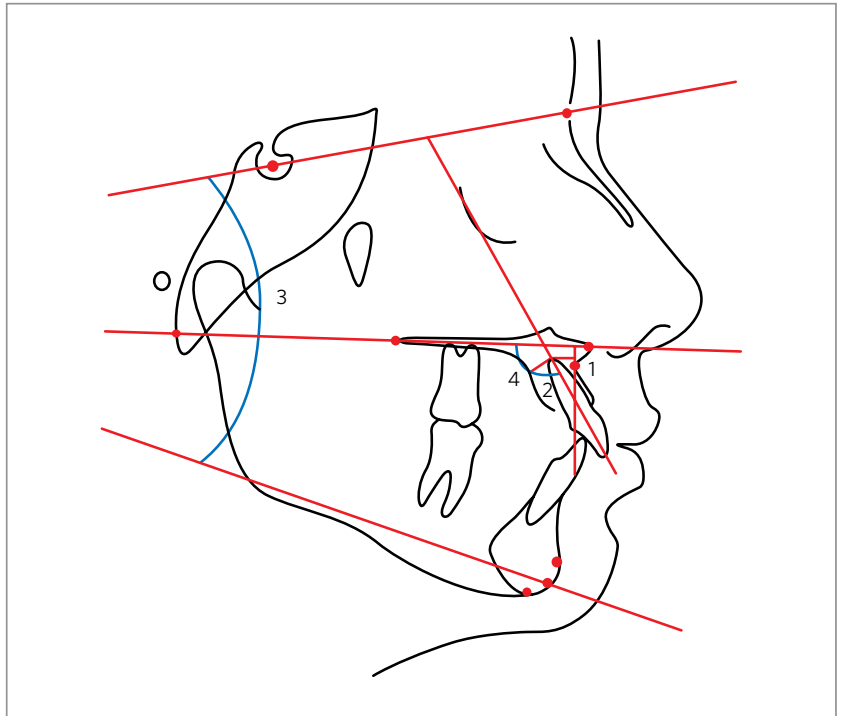

Figure 3 - Angular and linear measurements used in the study. 1- $A A B$; 2- PAB; 3- SN-GoGn; 4- 1. PP.

of the maxillary central incisors showed a palatal inclination, as measured by the reduction in angle 1.PP, being statistically higher in Group 2.

When observing the $\mathrm{AAB}$ value (Anterior Anatomical Barrier) and the PAB (Posterior Anatomical Barrier) in the two studied groups, it was noted that there was an increase in $\mathrm{PAB}$ and a reduction in $\mathrm{AAB}$, which confirmed the reduction in angle 1.PP (Tables 4 and 5), that is; the apexes of the maxillary central incisors were further from the posterior alveolar cortical bone and closer to the anterior alveolar cortical bone. It was also possible to note that there was a greater reduction of $\mathrm{AAB}$ in patients with vertical growth with statistical significance of $5 \%$ (Table 4). Whereas, in spite of a greater increase having occurred in $\mathrm{PAB}$ in Group $2\left(\mathrm{SN}-\mathrm{GoGn} \geq 43^{\circ}\right)$ in comparison with Group $1\left(\mathrm{SN}-\mathrm{GoGn} \leq 29^{\circ}\right)$, it showed no statistical significance (Table 5).

On this point, the two groups were related to each other, with the main object of finding a higher or lower amount of apical root resorption. Although no statistical significance was shown, surprisingly it was verified that the patients in Group $1\left(\mathrm{SN}-\mathrm{GoGn} \leq 29^{\circ}\right)$ presented a slightly higher rate of root resorption than the patients in Group $2\left(\mathrm{SN}-\mathrm{GoGn} \geq 43^{\circ}\right)$. To the contrary, it was expected that root resorption would occur to a greater degree in Group 2, in which the alveolar ridge was narrower (Table 6). 
Table 1 - Mean, standard deviation and level of significance of the SN-GoGn measurement for Groups 1 and 2 (in degrees).

\begin{tabular}{ccc} 
& \multicolumn{2}{c}{ SN-GoGn } \\
Mean & Group 1 & Group 2 \\
Standard deviation & 28.1667 & 45.2500 \\
Significance & 1.1690 & 1.9821 \\
\hline
\end{tabular}

* Significant at 5\%, ${ }^{\star \star}$ Significant at 1\%, ns - nonsignificant.
Table 2 - Mean value of initial $A A B$ (Anterior Anatomical Barrier), initial PAB (Posterior Anatomical Barrier) and level of significance between Groups 1 and 2 (in $\mathrm{mm}$ ).

\begin{tabular}{cccc} 
& Group 1 & Group 2 & Significance \\
\hline Initial AAB (mean) & 6 & 5.4 & ns \\
Initial PAB (mean) & 7.44 & 5.95 & $\star$ \\
\hline
\end{tabular}

* Significant at 5\%, ${ }^{\star \star}$ Significant at 1\%, ns - nonsignificant.
Table 3 - Mean, standard deviation, calculated " $t$ " and level of significance of Angle 1.PP for Groups 1 and 2 (in degrees).

\begin{tabular}{ccc|}
\hline & \multicolumn{3}{c|}{ 1.PP } \\
\hline Mean & -4.6875 & Group 2 \\
\hline Standard deviation & 7.5684 & -12.3000 \\
t-test (calculated) & & $-1.8056^{*}$ \\
Significance & & $*$ \\
\hline
\end{tabular}

* Significant at 5\%. ${ }^{\star \star}$ Significant at 1\%. ns - nonsignificant.
Table 4 - Mean, standard deviation, calculated " $t$ " and level of significance of $A A B$ (Anterior Anatomical Barrier) for Groups 1 and 2 (in degrees).

\begin{tabular}{|c|c|c|}
\hline & \multicolumn{2}{|c|}{$\mathbf{A A B}$} \\
\hline & Group 1 & Group 2 \\
\hline Mean & -0.7500 & -2.3500 \\
\hline Standard deviation & 1.6475 & 1.7803 \\
\hline t-test (calculated) & & \\
\hline Significance & & \\
\hline
\end{tabular}

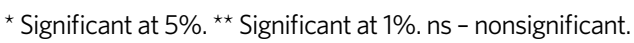

Table 5 - Mean, standard deviation, calculated " $t$ " and level of significance of PAB (Posterior Anatomical Barrier) for Groups 1 and 2 (in mm).

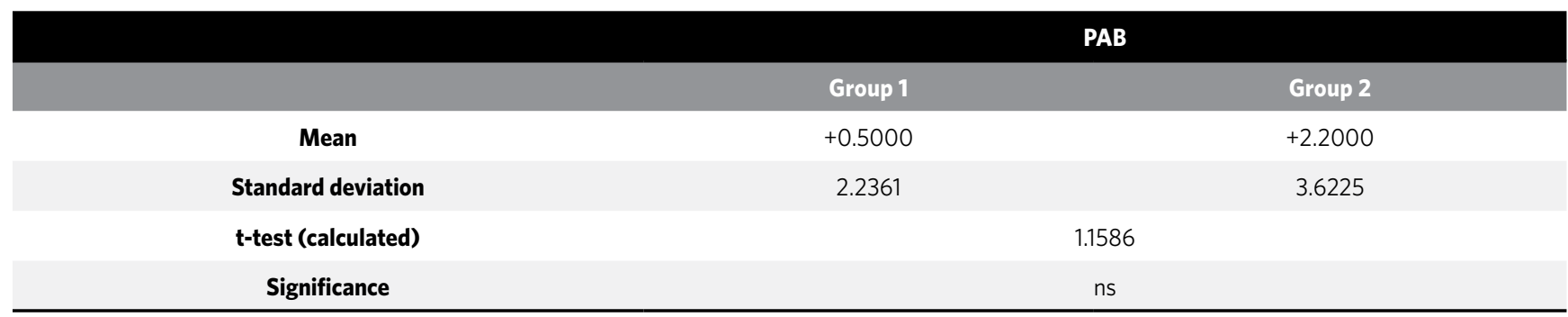

* Significant at 5\%. ${ }^{\star \star}$ Significant at 1\%. ns - nonsignificant.

Table 6 - Mean difference of measurements: 1.PP, AAB, PAB and mean value of the quantity of root resorption between Groups 1 and 2; inter-group differences, " $\mathrm{t}$ " test and level of significance.

\begin{tabular}{|c|c|c|c|c|c|}
\hline & \multirow{2}{*}{ Mean value 1.PP } & \multirow{2}{*}{ Mean value AAB } & \multirow{2}{*}{ Mean value PAB } & \multicolumn{2}{|c|}{ Mean value of the quantity of root resorption } \\
\hline & & & & Tooth 11 & Tooth 21 \\
\hline Group $1\left(\mathrm{SN}-\mathrm{GoG} \mathrm{n} \leq 29^{\circ}\right)$ & $-4.69^{\circ}$ & $-0.75 \mathrm{~mm}$ & $0.5 \mathrm{~mm}$ & $2.18 \mathrm{~mm}$ & $1.87 \mathrm{~mm}$ \\
\hline Group $2\left(\mathrm{SN}-\mathrm{GoG} n \leq 43^{\circ}\right)$ & $-12.30^{\circ}$ & $-2.35 \mathrm{~mm}$ & $2.20 \mathrm{~mm}$ & $1.68 \mathrm{~mm}$ & $1.45 \mathrm{~mm}$ \\
\hline Difference between groups & $7.61^{\circ}$ & $1.65 \mathrm{~mm}$ & $1.85 \mathrm{~mm}$ & $0.5 \mathrm{~mm}$ & $0.42 \mathrm{~mm}$ \\
\hline t-test & -1.8056 & -1.9572 & 1.1586 & $-0.52 \mathrm{~mm}$ & $-0.47 \mathrm{~mm}$ \\
\hline Statistic (level of significance) & * & * & ns & ns & ns \\
\hline
\end{tabular}

* Significant at 5\%, ${ }^{\star \star}$ Significant at 1\%, ns - nonsignificant. 


\section{DISCUSSION}

The literature reports that patients with excessively vertical growth (long face pattern) have a much narrower alveolar bone in the anterior region of the maxilla than patients who present horizontal growth (short face pattern). ${ }^{4}$

Following this line of reasoning, one supposes that patients with vertical growth (Group 2 - long face pattern), because they have a narrower bone, would be more likely to have their maxillary central incisors roots in contact with the alveolar cortical bone, in cases treated with extraction of premolars, and could consequently present a larger amount of apical root resorption.

The literature points out the importance of delineating the limits of orthodontic treatment in adults; ${ }^{4}$ therefore, researchers have determined the thickness from the anterior alveolar bone to the apex of the incisors in the maxillary and mandibular arches, because these measurements may represent the anatomical limits of tooth movement. They observed the thinness of the alveolar bone in both the buccal and the lingual regions of the mandibular incisors and the lingual region of the maxillary incisors in patients with vertical growth, ${ }^{4}$ confirmed by this study, which presented a narrower alveolar bone posterior to the apex of the maxillary incisor for individuals with a long face pattern.

There were no statistically significant anatomical differences between dolichocephalic and brachycephalic individuals at the alveolar bone level of the anterior region in the different types of Class I, II and III malocclusions. ${ }^{1}$ This study is in agreement with Assis ${ }^{1}$ with regard to the distance from the buccal cortical bone to the apex of the maxillary incisor, which showed no signifi- cance for the groups with vertical and horizontal growth. Similar results were found in other studies, ${ }^{6,7}$ which suggested that the morphology of the dentoalveolar complex was not a significant factor in the etiology of external apical root resorption.

Therefore, with the methodology used in this sample, it was evident that there were no correlations between the proximity of the cortical alveolar bone to the roots of maxillary central incisors and the rate of root resorption of these teeth. Other studies with larger samples may perhaps show different results from those found in this study.

\section{CONCLUSION}

Based on the analysis of the results obtained, based on the literature and considering the sample used in this research, it could be concluded that:

1) The distance from the posterior alveolar bone to the apex of the maxillary incisor (PAB) was statistically significant between Groups 1 and 2, that is; patients with excessively vertical growth (Group 2 - $\mathrm{SN}-\mathrm{GoGn} \geq 43^{\circ}$ ) have a narrower palatal alveolar bone in the anterior region of the maxilla than patients who present horizontal growth (Group 1 - SNGoGn $\leq 29^{\circ}$ ), while the distance from the buccal cortical bone to the apex of the maxillary central incisor (AAB) showed no significance for Groups 1 and 2.

2) Surprisingly, the patients in Group 1 (SN-GoGn $\leq 29^{\circ}$ ) presented a slightly higher rate of root resorption than patients in Group $2\left(\mathrm{SN}-\mathrm{GoGn} \geq 43^{\circ}\right)$, nevertheless, the values obtained showed no statistical significance between the studied groups, leading to the conclusion that there was no correlation between the proximity of the cortical alveolar bone to the roots of maxillary central incisors and the rate of root resorption of these teeth.

\section{REFERENCES}

1. Assis AMA. Importância do processo alveolar da região anterior superior e inferior na limitação do tratamento ortodôntico [monografia]. Varginha (MG): Associação Brasileira de Odontologia Regional de Varginha; 2003.

2. Consolaro A. Reabsorções dentárias nas especialidades clínicas. 2a ed. Maringá: Dental Press; 2005.

3. Garcia RM, Claro CAA, Chagas RV, Almeida G. Espessura do processo alveolar da região anterior da maxila e mandíbula em pacientes com discrepância óssea antero-posterior. Rev Dental Press Ortod Ortop Facial. 2005;10(5):137-48.

4. Handelman CS. The anterior alveolus: its importance of in limiting orthodontic treatment and its influence on the occurrence of iatrogenic sequelae. Angle Orthod. 1996;66(2):95-110.
5. Horiuchi A, Hotokezaka H, Kobayashi K. Correlation between cortical plate proximity and apical root resorption. Am J Orthod Dentofacial Orthop. 1998;114(3):311-8.

6. Mirabella AD, Arthur J. Risk factors for apical root resorption of maxillary anterior teeth in adult orthodontic patients. Am J Orthod Dentofacial Orthop. 1995;108(1):48-55

7. Otis LL, Hong JS, Tuncay OC. Bone structure effect on root resorption. Orthod Craniofac Res. 2004;7(3):165-77.

8. Taithongchai R, Sookkorn K, Killiany DM. Facial and dentoalveolar structure and the prediction of apical root shortening. Am J Orthod Dentofacial Orthop. 1996;110(3):296-302 